\title{
Fluctuation Electron Microscopy of Shungite, a Disordered Natural Carbonaceous Material
}

\author{
M. M. J. Treacy ${ }^{*}$, A. Rougee*, and P.R. Buseck ${ }^{* *}$ \\ *Dept. of Physics and Astronomy, Arizona State University, Tempe, AZ 85287 \\ ${ }^{* *}$ Depts. of Geological Sciences and Chemistry/Biochemistry, Arizona State University, \\ Tempe, AZ 85287
}

Fluctuation electron microscopy has received much attention as a technique for the study of amorphous synthetic materials such as semiconductors, metallic glasses and synthetic carbons $[1,2]$. However, the technique has not yet caught on as a tool for studying the rich and varied forms of disorder that occur in other materials, including those found in nature. Carbon is known to form in a wide range of structures. Here we report on a somewhat unusual form, called shungite, which forms naturally in the Shunga region of Karelia, Russia. It is black, glassy, and resembles anthracite but is significantly denser. Studies of its structure using TEM, x-ray diffraction and ${ }^{13} \mathrm{C}$-nuclear magnetic resonance (NMR) show that it is highly disordered [3,4]. However, the disorder is inhomogeneous, varying significantly among different samples and even within a sample. Shungite excited much attention when it was discovered that some samples contain $\mathrm{C}_{60}$ buckyballs [5]. Since then others have observed fullerenes in both shungite and other terrestrial and extra-terrestrial minerals using mass spectroscopic methods.

The rich disorder within shungites makes them natural candidates for study by fluctuation electron microscopy. Shungite samples were prepared for TEM by crushing in a mortar and pestle. Shungite is brittle, and the best samples were obtained by tapping small grains with a vertical blow of the pestle, avoiding shearing motion. Samples were deposited on lacey carbon films and examined in a LEO 912 TEM at $120 \mathrm{kV}$. A $10 \mu \mathrm{m}$ objective aperture was used for images, corresponding to $\sim 1.0$ $\mathrm{nm}$ point-to-point resolution. The microscope was controlled using a Digital Micrograph ${ }^{\mathrm{TM}}$ script. Series of tilted dark-field images were obtained from sample areas by tilting the beam from $Q=-14$ $\mathrm{nm}^{-1}$ to $Q=+14 \mathrm{~nm}^{-1}$ along both the $x$ and $y$ tilt directions. $Q$ is a measure of the beam tilt, and corresponds to the reciprocal of the crystal spacing that can Bragg reflect at that angle. $Q$ therefore probes structural spacing within the sample. Beam tilt was calibrated using a polycrystalline aluminum sample. The script adjusted exposure times to maintain about 500 counts per pixel to keep shot noise uniform. The normalized image variance was computed after noise from $\mathrm{x}$-rays was removed. Two images from a typical series are shown in Fig. 1. Speckle that is characteristic of amorphous materials is evident near $Q=3 \mathrm{~nm}^{-1}$ (Fig. 1a). However, as is evident at higher $Q$ (Fig 2b), the sample thickness is not uniform, and this contributes to the measured variance at high $Q$.

Fig. 2 shows typical normalized variance plots as a function of scattering vector from three different regions of the same shungite sample. We find that this sample is heterogeneous, with some areas appearing amorphous, whereas other areas show evidence of medium-range graphitic ordering. No direct evidence of ordered fullerenes has been found to date. Future work will involve comparing the shungite results with normalized variance data from well-calibrated amorphous carbons, nanodiamonds and carbon blacks as well as disordered buckyball samples.

The authors thank Karl Weiss and Laurence Garvie for assistance with sample preparation. 


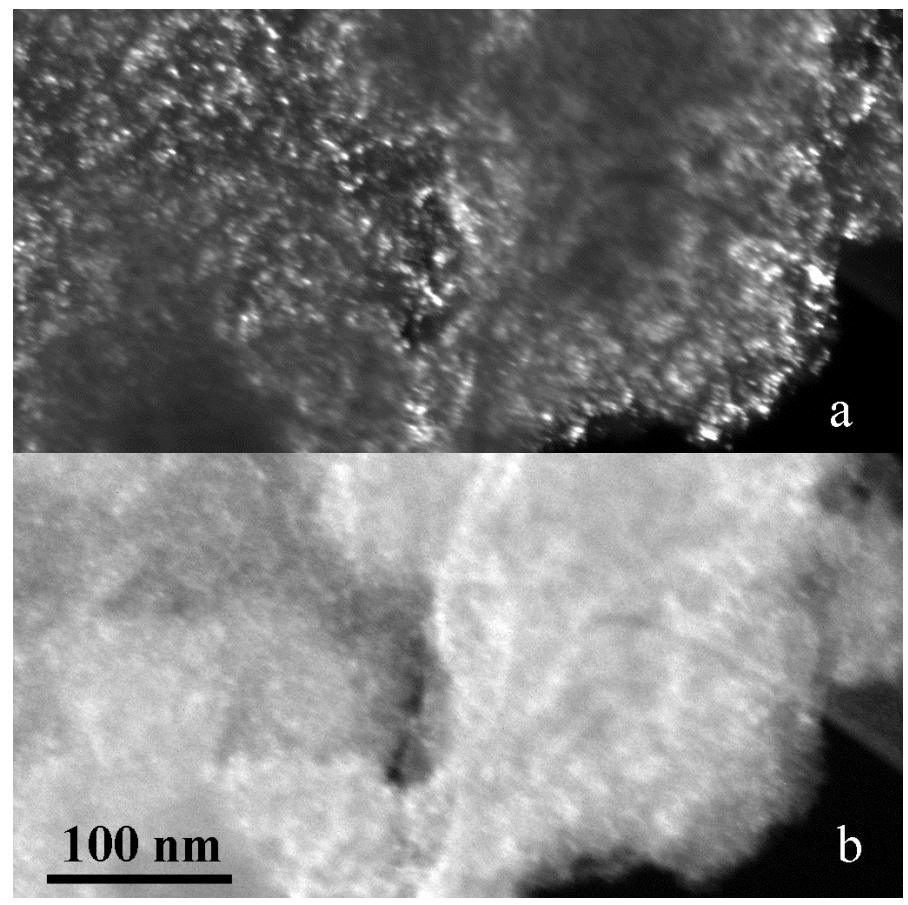

Fig. 1. Tilted dark-field images of shungite, area 1. a) Image taken near $Q=$ $3 \mathrm{~nm}^{-1}$, close to where the graphite 001 reflections are expected. The speckle shows that no extended graphitic regions are present. b) Same area near $Q=10$ $\mathrm{nm}^{-1}$. Here the image is less speckly, but pronounced intensity changes are evidence of large thickness variations.

Fig 2. Normalized variance of a series of tilted dark-field images from three regions of a shungite sample. Area 2 is of fairly uniform thickness; and shows the signature of amorphous carbon. Areas 1 and 3 are less uniform in thickness. The rise at high $Q$ is primarily from the non-uniform specimen thickness across the image. The peaks at 7 $\mathrm{nm}^{-1}$ have not been identified, but they are not due to graphitic carbon [2]. No variance signature corresponding to buckyball fullerenes has yet been identified.

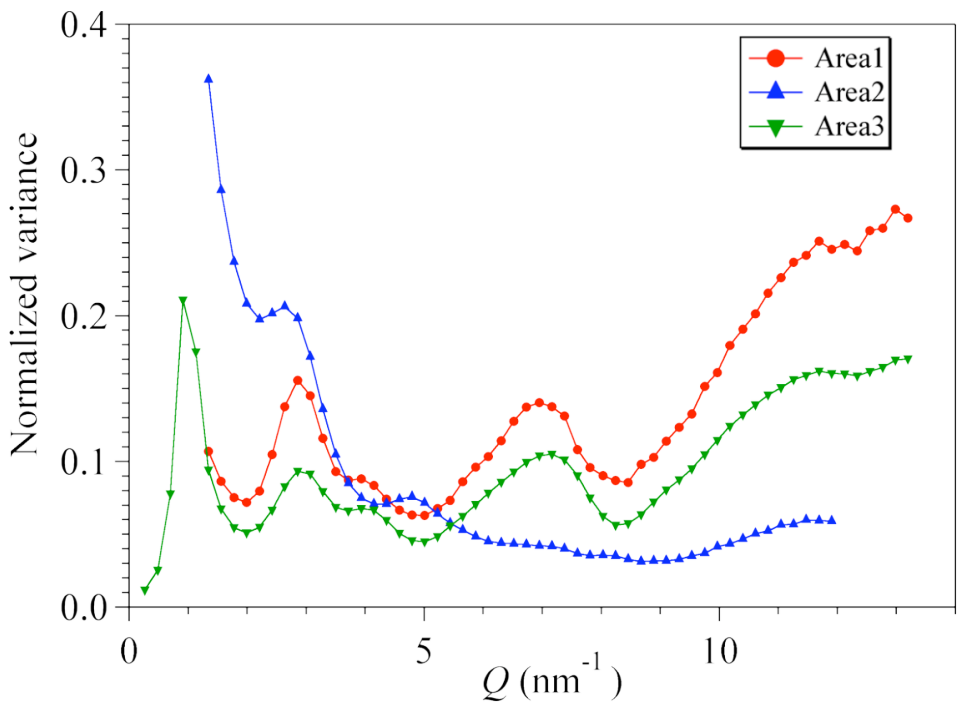

\section{References}

1. M. M. J. Treacy, J. M. Gibson, L. Fan, D. J. Paterson and I. McNulty, Rep. Prog. Phys. 68 2899-2944 (2005).

2. X. Chen, J. P. Sullivan, T. A. Friedman and J. M. Gibson, Appl. Phys. Lett. 842823 (2004).

3. V.V. Kovalevski, P.R. Buseck, and J.M. Cowley, Carbon, 39, 243 (2001).

4. Parthasarathy, G., Srinivasan, R., Vairamani, M., Ravikumar, K., and Kunwar, A.C., Geochimica et Cosmochimica Acta, v. 62, p. 3541-3544 (1998)

5. P.R. Buseck, S.J. Tsipursky and R .Hettich, Science, 257, 215 (1992). 